\title{
Book review: Oxford textbook of oncology, 3rd edition
}

\author{
Editors: Kerr DJ, Haller DG, van de Velde CJ, Baumann M \\ ISBN: 978-0-19-965,610-3 date of publication 2016 cost $£ 230.00$
}

\author{
Andrew Davies ${ }^{1}$ \\ Received: 12 October 2016 / Accepted: 26 October 2016 / Published online: 3 November 2016 \\ (C) Springer-Verlag Berlin Heidelberg 2016
}

It has been 15 years since the second edition of this eminent text book was published. In the interim, there have been significant advances in oncology, and so it is somewhat surprising (but welcome) to see that the third edition of the text book is much more compact than the second edition (i.e. one volume of approximately 1000 pages versus two volumes of approximately 3000 pages). Nevertheless, the third edition contains all necessary information for the non-specialist aiming to be up-to-date with the said advances in oncology.

The first section ("Hallmarks of cancer") is especially useful, since it discusses the complex biology of cancer, which in turn explains the rationale for new treatment strategies. The fifth section ("Support for the cancer patient") includes sections on Supportive and Palliative care, as well as Quality of Life and Cancer Survivorship and Rehabilitation. These topics have become increasingly important in oncology, and were hardly mentioned in the previous edition. The latter disease orientated chapters contain good overviews of the current management of specific tumour types. However, the publishers/editors have been mindful of the rapid developments in the field of oncology, and so the accompanying online version of the textbook will be updated on a regular basis (to reflect changes in management). Purchasers of the textbook will have free access to the online textbook for a period of 12 months.

The second edition spent most of its time gathering dust on a shelf in my office. The third edition is much more user friendly, and I anticipate that I will use it much more than its predecessor. I would recommend anyone considering buying an oncology textbook, and particularly those who work in oncology "support services", to consider this textbook as it is well set out, easy to read, easy to comprehend and covers all of the important aspects of modern day oncology.

Andrew Davies

adavies12@nhs.net

1 Royal Surrey County Hospital/St. Luke's Cancer Centre,

Guildford, UK 